The nodules are surrounded by a fibrous band of compressed liver tissue. In some places this fibrous band shows lacunae containing degenerated liver cells. The larger nodules are packed with large round or irregular cells with large nuclei, some areas resembling

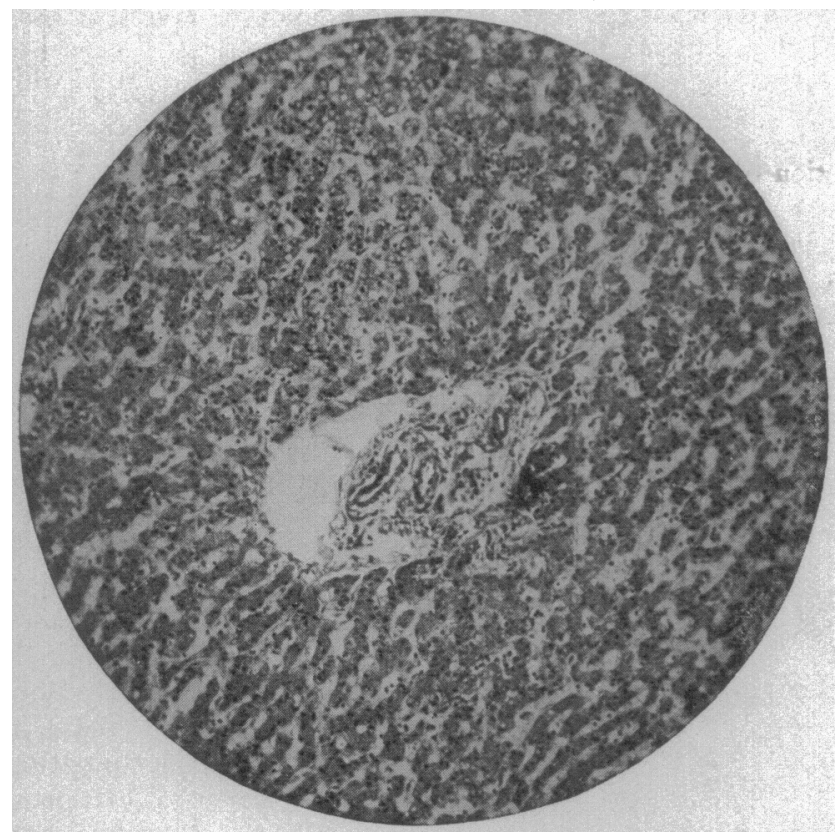

Fig. 6.-Larger vessels show swollen endothelium, smaller ones infiltration; $\times 100$

myxosarcoma. Giant cells and polymorphonuclear leukocytes are also seen in some areas. Hemorrhagic areas are also noticeable in some fields.

1726 M Street N.W.

\section{SOME UROLOGIC ASPECTS OF DERMOID CYSTS *}

\section{WILLIAM C. QUINBY, M.D. BOSTON}

Although in gynecologic and surgical literature and textbooks the varying clinical manifestations caused by dermoids are well known and frequently noted, it is only in rare instances that they are brought to the attention of the urologist. Such growths may at times, however, give rise to marked disturbance and involvement of the urinary tract, and it is to emphasize this fact that the following case histories are reported:

\section{REPORT OF CASES}

CASE 1 (7888).-Dermoid cyst of the vesicovaginal septum, causing ulceration of the bladder with profuse hematuria.

History.-A married woman, aged 25, entered the Peter Bent Brigham Hospital complaining of hematuria. She had had two children without any complications of delivery. Nine years before her entrance into the hospital, there had occurred occasional attacks of urgent urination. About one year previously, these attacks had become more severe, and at this time undue frequency of urination also appeared There was a feeling at the end of micturition as if she still should pass water, but she was unable to do so. There was never any pain in the bladder. About two months before her admission, hematuria began, and at times the blood was

* From the Urological Clinic of the Peter Bent Brigham Hospital. * Read before the Section on Urology at the Seventieth Annual Session of the American Medical Association, Atlantic City, N. J., June, 1919. seen in clots. More tecently, the bleeding had increased definitely in amount.

Examination.-The general physical examination disclosed nothing abnormal. Vaginal examination revealed a moderate sized cystocele and a slight lateral tear of the cervix uteri. Otherwise, the pelvic organs were normal.

Examination of the bladder by use of the cystoscope showed it to be normal except for an area $2.5 \mathrm{~cm}$. in circumference situated on the left aspect of the trigon, just inside the internal urinary meatus. This area was raised above the level of the bladder, and the mucous membrane was irregular, eroded and ulcerated. In one or two areas there were hemorrhagic spots, evidently the source of the bleeding. In other areas there were extensive phosphatic deposits. The ureters were uninvolved and normal in appearance. Normal urine, free from blood, came from each. It was felt that this area in the floor of the bladder was probably neoplastic in origin because of (1) its long duration without other signs than those of bleeding, and (2) its tendency to necrosis with a deposit of lime salts. It seemed wise to advise excision of the area.

Operation.-The abdomen was opened, Dec. 24, 1917, by a vertical incision in the midline extending from the pubes to within $3 \mathrm{~cm}$. of the umbilicus, the patient being in the high Trendelenburg position. Through this incision the intestine was walled off by gauze packs and the uterus drawn backward. The peritoneum was incised transversely at the level of its reflection onto the bladder, and the bladder separated from the anterior surface of the uterus by blunt dissection. There were no evident areas of neoplasm in this region. The bladder was then opened by a vertical incision made from above downward on its posterior wall, and the ulcerated area inspected. This was found to be considerably raised above the general surface as previously described. On ocular

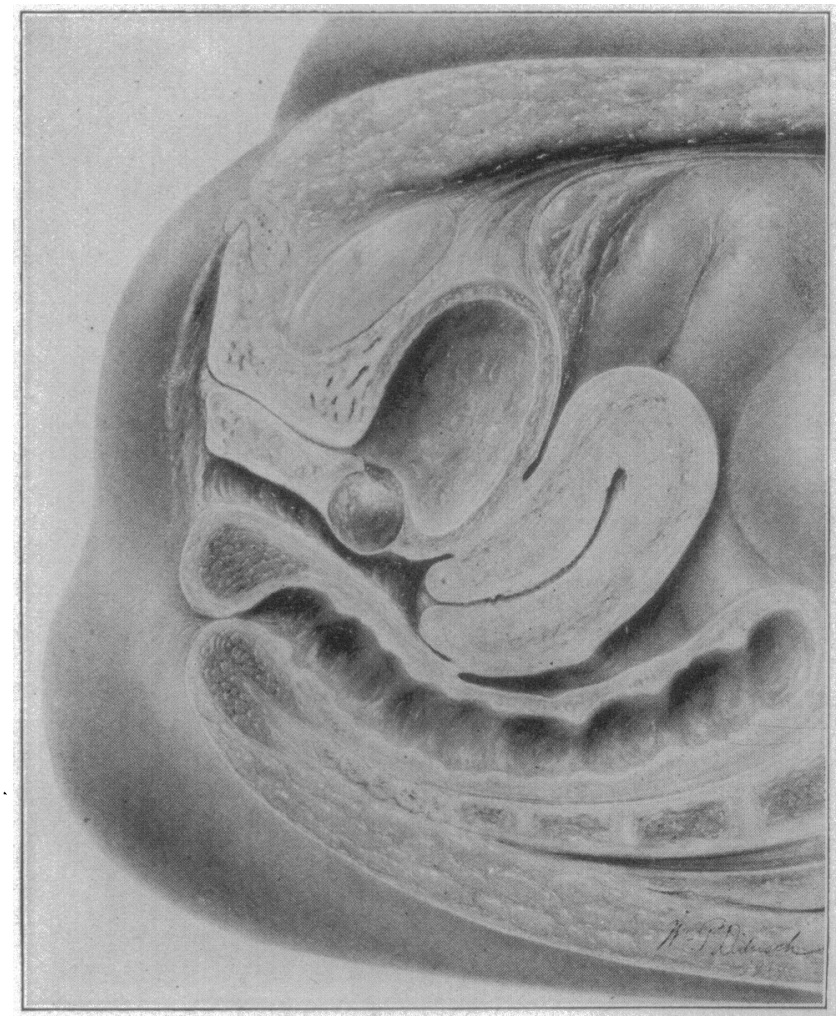

Fig. 1 (Case 1).-Dermoid of vesicovaginal septum causing ulceration of bladder.

inspection, however, it did not look especially malignant. By extending the incision in the bladder wall downward, and by the aid of sutures placed over the edges of the cut to aid in traction, it was possible to surround the ulcerated area by an elliptic incision, cutting through all thicknesses of the 
bladder. The area in question was thus excised. The bladder was repaired by interrupted stitches of fine catgut in a double row. It was found impossible to place accurately the stitches nearest the urinary meatus, and therefore this was left to be approximated from the vaginal approach After closure of the bladder as high as its vertex, the peritoneum was sutured. by a running catgut stitch, and a drain was left in the bladder to divert the urine. The abdomen was then closed in the usual manner by a continuous stitch for the peritoneum, interrupted stitches for the fascia, and silk for the skin.

The patient was then placed in the lithotomy position, and preparation was made to separate the anterior vaginal wall from the neck of the bladder. On following the transverse incision below the urethra, it was at once seen that what had previously been considered a cystocele was a dermoid cyst. This was excised and found to be filled with possibly 10 c.c. of material like yellow, thick cream. The wall of the cyst was excised and found to connect superiorly immediately with the area of the bladder which had previously been dissected. The bladder floor was closed over this incision by interrupted stitches, and afier being sure that the wall of the cyst was entirely removed, the anterior vaginal mucosa was sutured with interrupted $\mathrm{cll}$ rom ic catgut. A vaginal pack was put in place for twenty-four hours.

It is evident that we have here dealt with a chronic bleeding ulcer of the bladder, which was a complication of a dermoid cyst of the anterior vaginal wall, and was caused by it. Aithough the diagnosis was not made before operation, it seems probable that this condition in the bladder would have responded readily to no treatment other than excision.

Postoperative History.-The patient made an uninterrupted recovery and was discharged on the nineteenth day after the operation. At this time, cystoscopy revealed a perfect healing of the bladder floor.

A recent note from the patient states that she is entirely cured.

CASE 2 (10055).-Dermoid cyst of the ovary flling the true pelvis; communication with vault of bladder; severe cystitis.

History.-An unmarried woman, aged 43, entered the Peter Bent Brigham Hospital, complaining of dysuria. Except for the fact that the patient had had an attack of "peritonitis" when she was 23 years old, the past history was essentially negative. Until four months before entrance, she had been entirely well. She then noticed suddenly that urination was painful, and the condition had persisted ever since. There was moderate frequency of urination, and the urine was turbid. There was never any hematuria, and there were no attacks of fever. During the month before admission, the bowels had become exceedingly costive. The patient also noticed that she was losing weight. During six weeks preceding her admission to the hospital, the dysuria and frequency became more marked, and she was finally required to urinate at least five or six times at night. There had been a dull, intermittent, suprapubic pain for two weeks, and this was not relieved by emptying the bladder. Pain in the lower abdomen, on walking, had also been a late development.

Examination.-The patient was poorly developed and nourished. The only abnormality found was a mass above the pubes, rounded in outline with its base below, extending half way up to the umbilicus. Its greatest width was $11 \mathrm{~cm}$. Palpation revealed a firm, moderately tender tumor which did not disappear on emptying the bladder. There was no tenderness in either costovertebral angle. When this mass was palpated by rectum, it was found to fill the whole pelvis and to cause marked downward dislocation of the uterus. On account of extreme tenderness it was impossible to determine the relations of this mass definitely.

The urine was cloudy, with a specific gravity of 1.017 , and in the sediment were many leukocytes and small round cells. No blood or casts were found.

On cystoscopy, the bladder was found to be of slightly diminished capacity, and contained a considerable amount of thick, ropy pus. There was considerable infection in the region of the trigon. In the posterior upper portion of the bladder wall, just below its vertex, was seen an area, about 1 by $2 \mathrm{~cm}$. in size, showing sloughy and irregularly hyperemic elevations and depressions, and surrounded by bullous edema. It was considered to be uncertain whether this area in the vault represented a definite penetration of the bladder or whether it was merely due to circulatory changes in the wall, due to adhesions formed between it and the pelvic mass.

Operation.-March 6, 1919, with the patient in the Trendelenburg position, a linear incision was made from above the symphysis to the umbilicus, in the median line. The omentum was found firmly adherent in a large mass low just behind the pubes in the region of the vault of the bladder. Below this adherent omentum was a tumor mass with a roughly nodular surface, semifluctuant. On further exploration, this mass was found to be intimately adherent to all the pelvic structures. It was necessary to resect the omentum in order to lift it upward. This was done, cutting after tying off with sutures passed on needles. It was then found that the mass represented the right ovary, and that it was a dermoid cyst containing pultaceous material and hair. After enucleation from the posterior surface of the right broad ligament, firm adhesions to the sigmoid were encountered These were cut after ligation and the mass lifted upward. On the extreme left it was not adherent, but above the fundus of the uterus and in front it was densely adherent to the bladder, and in separating these adhesions the bladder was inevitably entered, with the escape of some of its contents into the pelvis. The bladder was sutured, after removal of 
the cyst, as well as might be, although it was quite impossible on account of the absence of peritoneum to make a satisfactory separation of the bladider from the peritoneal cavity. Following removal of the cyst, the pelvis was drained with one large cigaret tube, and the bladder drained by a selfretaining catheter in the urethra. The operation was exceedingly difficult on account of the density of the adhesions, and its duration was two hours. The patient's condition, however, at the end of the operation, was quite satisfactory.

Subsequent Condition.-Six days after the operation the following note was made:

"Since operation the patient has been showing increasing signs of intestinal obstruction. She has vomited at irregular intervals, which have grown more frequent. She has been quite unable to retain any nourishment and there have been frequent intervals when the pulse has been poor. She has had no fever at any time. The bladder condition has been fairly satisfactory. The catheter has drained well enough for the most part, although two days ago there were signs of leakage around the abdominal drain, and this was therefore replaced by a small sized tube. The output of urine has been only moderate in amount, but seems to have been sufficient. Today the abdomen is tensely distended, but there is no dulness to be found in either flank, although the patient is vomiting green bile. She is very weak and has typical abdominal facies. It is evident that in spite of repeated enemas and stomach washouts she is not making progress. Secondary operation is therefore necessary to relieve probable obstruction."

Second Opcration.-March 13, 1919, under light anesthesia, the old incision was opened and the abdomen explored. No collapsed bowel could be found, all the bowel, large as well as small, being markedly distended. There was no purulent peritonitis present, although over the coils of the pelvis there were occasional bits of fibrin with adhesion. One coil of ileum was quite markedly kinked deep in the pelvis on the right side, and, after release, its gas was heard to move freely through the intestine. All coils of bowel which had become adherent in the site of the previous operation were freed, and a Mixter tube was quickly sewed into the cecum. This was led out from the abdomen through a small incision in the right lower quadrant. The pelvis was again drained with a deep cigaret wick and the wound closed in the usual manner.

From the second operation the patient did not rally, and death occurred about thirty-six hours later.

Postmortem Examination.-Paralytic ileus following fibrinous peritonitis was found at necropsy, together with necrosis of the posterior wall of the bladder, with cystitis, and cellulitis of the perivesical tissues.

This condition was undoubtedly the result of partial rupture of the adherent dermoid during its removal, with some soiling of the pelvis. The content of dermoid cysts is well known to have a toxic proteolytic action on any fresh tissue with which it comes in contact. In the peritoneum this action is marked and results in a nonbacterial adhesive peritonitis. Leakage of urine through the sutured area in the bladder probably played a very minor part in causation of the peritonitis, if leakage occurred at all, for at the second operation the suture seemed to be holding firmly

Fxamination of Dermoid Cyst.-Gross description: The specimen consisted of a cyst about $7 \mathrm{~cm}$. in diameter. The surface was rather bright red. There were numerous injected blood vessels. It felt boggy. Its contents consisted of a large mass of brownish hair mixed with a grayish yellow material about the consistency and general appearance of thin clay. The wall varied in thickness from 2 to $4 \mathrm{~mm}$. It was quite fibrous, and anteriorly was covered with a very vascular granulation tissue on which was a layer of yellow necrotic tissue about 1 to $2 \mathrm{~mm}$. in thickness. This could be stripped off, exposing a very vascular, roughened surface. From one place over an area about $2 \mathrm{~cm}$. wide the growth of hair seemed to spring, the hair being intimately adherent to the wall by a red, fibrous pedicle. There were two or three areas where the wall was thickened and quite firm, and on section it was pale white and with the consistency of cartilage. A frozen section of this portion of the wall showed it to consist chiefly of a hyalin framework in which there were numerous leukocytes and necrotic cells. A frozen section in another portion of the wall was seen to consist chiefly of granulation tissue. Smears of the yellow exudate showed necrotic leukocytes, necrotic cells and gram-negative bacilli.

Microscopic Report: There were three sections from various parts of the cyst wall. Lining the cyst was a thick layer of granulation tissue containing numerous giant cells and occasional small areas of necrosis. Beneath this layer the wall was composed of dense fibrous tissue which in great part was of a pale, hyalin appearance. There were numerous small abscesses in the wall with intense polymorphonuclear reaction and areas of necrosis. Many of the smaller blood vessels showed the changes of obliterative endarteritis and perivascular mononuclear infiltration.

Diagnosis: The diagnosis was: a dermoid cyst, with acute and chronic inflammation of the wall.

A third case illustrating in striking manner the action of dermoid cysts when causing renal colic is found in the recent report of Fagge. ${ }^{1}$

CASE 3.-History.-Nurse H., aged 27, complained one evening of dull "stomach-ache," which she had endeavored to treat with castor oil, but without success. The history she gave was that she had had scarlet fever in childhood, but no other illness of importance. The recent pain had been at first a diffuse abdominal pain. She had taken a dose of castor oil, and then, as the first dose had made her sick, she had taken another.

The physician who first saw her regarded the case as one of so-called gastritis, and gave her an anodyne; this alleviated the pain, and she slept fairly well. By the following evening, however, the pain had returned; it originated in the left loin, radiated to the left subcostal region, around the waist, and (a little later) toward the groin. It was of a colic-like nature, and accompanied by nausea, though not by actual vomiting. No tumor could be felt in the renal region, or elsewhere, and the urine was reported by the sister of the nurse's sickroom to be normal on chemical examination

Renal colic due to calculus was suspected, and a roentgenogram was taken, which cleared up the diagnosis. Two teeth were to be seen very plainly at the level of the pelvic inlet-the root-canals could distinctly be seen in the original plate. Since the patient had not swallowed any teeth, it was evident that she was suffering from an ovarian dermoid cyst. Moreover, roentgenograms of the kidneys gave no evidence of calculi, so that the cyst was probably the cause of the symptoms, and not merely incidental.

The patient was transferred to a surgical ward. While awaiting the visit of the surgeon in charge, a sudden change occurred in her condition. She began to vomit violently and became distended and tympanic; in short, she showed not only signs of pressure on the ureter, but also those of intestinal obstruction. It should be remarked that prior to this attack of obstruction, she had suddenly passed an unusually large quantity of urine, suggesting that the renal symptorns which had predorninated at the early stage of her illness were due to hydronephrosis-closed at first and finally opened.

Operation and Result.-In view of the sudden change in the patient's condition, a laparotomy was performed with the least possible dclay; it was found that there were two ovarian cysts, firmly impacted in the pelvic brim. Both were multilocular, and contained hair and pultaceous material of the usual kind. The teeth were found in the right cyst, while the left cyst had evidently pressed on the pelvic colon and caused the obstructive symptoms. The left ureter showed no external signs of obstruction, but as some hours had elapsed since the discharge of urine, it is not surprising that no such sign was seen, and the absence of any such indication in no way controverts the diagnosis of hydronephrosis. The pelvic colon showed the usual signs of recent moderate

1. Fagge, C. H.: Bilateral Ovarian Dermoid Cysts Simulating Renal Colic, Brit. J. Surg. 6: 468 (Jan.) 1919. 
obstruction. Both cysts were excised. No trace of ovarian stubstance or of supernumerary ovaries could be seen macroscopically. Recovery was uneventful.

The further history of the case is not at present ascertainable, but it seems probable that complete amenorrhea will soon set in, with all the ordinary train of physical and psychic changes, for the girl's appearance, prior to the operation, was stuch as to suggest that her supply of ovarian internal secretion had long been deficient.

The main interest of the case centers in the unusually complete simulation of renal trouble at the start, and then the sudden transformation into a case of intestinal obstruction; and also in the rapid and certain aid to diagnosis afforded by the roentgen rays.

The teeth in the right dermoid were about as long and as thick as adult canines; the crowns were slightly flattened, and showed several little cingula near the top. Each tooth had a single root, though the apex of one of them was a little bent to one side. No trace of dental or bony matter was found in either cyst beyond these two teeth, though there were a few partially calcified areas in the débris.

\section{COMMENT}

Such cases as these are illustrations of the fact that a dermoid cyst, frequently quite benign, may later take on characters of growth causing marked adhesion and even penetration into an adjacent viscus. When the bladder is thus involved or the ureters occluded by pressure, a knowledge of such characteristics by the urologist is all important.

\section{ABSTRACT OF DISCUSSION}

Dr. Hugh H. Young, Baltimore: A man came to the dispensary of the Johns Hopkins Hospital some time ago bringing with him a small calculus that he had passed in his urine. It was attached to a hair. We cystoscoped him and found another calculus on the anterior wall of the bladder. It, too, was attached to a hair. As the patient was shaking, it would move back and forth like a pendulum, and it was very easy to trace it to a little opening on the anterior wall of the bladder, which very evidently had some pocket. He was not willing to go into the hospital then, therefore we caught this calculus with a cystoscopic rongeur and removed it, thinking that it might possibly cure him. In a few months he returned with another hair calculus. I did a suprapubic cystotomy and removed a pocket in the anterior wall which contained a very small amount of hair. Apparently he has been cured by this simple procedure.

Dr. Arthur L. Chute, Boston: About two years ago I saw a case of dermoid of the ovary that had ruptured into the bladder. The patient, who had been having urinary distress with an infected urine, showed, on cystoscopic examination, a bladder that contained great masses of hair. A mass as big as my fist, filled with hair, was removed abdominally and the opening into the bladder was closed. The patient made a good recovery.

Influenza in the Philippines.-In the Revista Filipina de Medicina y Farmacia, Dr. Eugenio Hernando, the chief of the provincial division of the sanitary service of the Philippines, reviews the epidemic of influenza that occurred in the Philippines from June to December, 1918. The total number of deaths caused by the epidemic was 70,513 . It is estimated that the disease attacked 40 per cent. of the total population of about $7,000,000$, the mortality being about 2.5 per cent. of those attacked. The epidemic really began in June although it did not assume severity until October. The groups of ages that suffered most were those between 10 and 29 years. The disease did not seem to be imported, since cases were occurring before any ships arrived from infected countries, although after the importation of cases it assumed a more severe form. The June epidemic seemed to confer a certain degree of immunity during the second outbreak of the disease in October.

\section{THE SIMULATION OF MENINGITIS BY INFLUENZA}

FRED H. STANGL

Resident Pathologist, Cook County Hospital

\section{CHICAGO}

In reports of the pandemic of influenza and its complications, reference has been made to toxemia and to the symptoms of shock and meningitis. Thus, Keeton and Cushman ${ }^{1}$ mention the delirium observed early as well as late in the disease, and liken the shock to that following operations. They noted the engorged conjunctival blood vessels, so constantly seen in the acute exanthems, in a number of the patients suffering from influenza and pointed out the difficulty not inf requently encountered in differentiating influenza from meningitis.

Evidence of this systemic infection is also present in statements regarding toxemia and delirium in the reports made by Friedlander and his co-workers, ${ }^{2}$ and by Synnott and Clark. ${ }^{3}$ Strouse and Bloch ${ }^{4}$ mention one case, in the 500 studied clinically by them, at first suggestive of meningitis but with the spinal fluid unchanged except for increased tension. Nervous manifestations were observed in the patients suffering from influenzal pneumonia and also from uncomplicated influenza by Lyon and others ${ }^{5}$ at Camp Upton. More recently $\mathrm{Neal}^{6}$ has made this feature of influenza the subject of special consideration. She reports twenty instances in which she observed meningeal symptoms, headache, stiffness of the neck, positive Kernig sign, and extreme apathy, in patients convalescing from attacks which clinically appeared to be influenza. The spinal fluids in her patient's were negative in chemical and cytologic constituents with rare exceptions. Bloomfield and $\mathrm{Harrop}^{7}$ and Alexander ${ }^{8}$ have referred to the evidence of systemic infection in cases of influenza and have likened the onset to that of the acute infections and acute exanthems with constitutional reactions, such as headache, chills, fever, general aching, malaise, prostration, anorexia, nausea and vomiting. Generalized infection in influenza is also referred to by Le Count, ${ }^{9}$ who states that it is difficult to ascribe death to the small amount of lung tissue actually pneumonic in many cases of influenzal pneumonia.

With these matters in mind we have reviewed again the records studied by Keeton and Cushman with the addition of those which have accumulated since their report, 3,400 cases in all, and find that nearly 1 per cent. of these patients had symptoms suggestive of meningitis or cerebral involvement. They varied

1. Keeton, R. W., and Cushman, A. B.: The Infuenza Epidemic in Chicago, A Type of Toxemic Shock, J. A. M. A. 72:1962 (Dec. 14) 1918

2. Friedlander, Alfred; McCord, C. P.; Sladen, F. J., and Wheeler, G. W.: The Epidemic of Influenza at Camp Sherman, Ohio, J. A. M. A. 71: 1652 (Nov. 16) 1918 .

3. Synnott, M. J., and Clark, Elbert: The Influenza Epidemic at Camp Dix, J. A. M."A. 71:1816 (Nov. 30) 1918.

4. Strouse, Solomon, and Bloch, Leon: Notes on the Present Epidemic of Respiratory Disease, J. A. M. A. 71: 1568 (Nov. 9) 1918. 5. Lyon, I. P.; Tenney, C. F. and Szerlip, Leopold. Some Clinical Observations of the Influenza Epidemic at Camp Upton. J A. M. A. 72: 1726 (June 14) 1919.

6. Neal, Josephine B.: Meningeal Conditions Noted During the Epidemic of Influenza, J. A. M. A. 72: 714 (March 8) 1919.

7. Bloomfield, A., and Harrop, G. A.: Epidemic of Influenza Bull. Johns Hopkins Hosp. 30:1 (Jan.) 1919.

8. Alexander: Berl. klin. Wchnschr., 1918, No. 38; abstr. Deutsch. med. Wchnschr. 44: 1171,1918 9. Le Count, E. R.: The Pathologic Anatomy of Influenzal Broncho-
pneumonia, J. A. M. A. T2: 650 (March 1) 1919 . 